\title{
Avanços tecnológicos em oncologia: reflexões para a prática de enfermagem
}

\author{
Technological advances in oncology: reflections for the nursing practice
}

\author{
Silvia Regina Secoli, ${ }^{1}$ Kátia Grillo Padilha, ${ }^{2}$ Rita de Cássia Burgos de Oliveira Leite ${ }^{1}$
}

\begin{abstract}
Resumo
Este artigo tem como proposta tecer al gumas considerações sobre as incorporações tecnológicas em saúde, a hardware ou dura e a software ou leve, ambas importantes, essenciais e complementares para o diagnóstico e tratamento oncológico, como também refletir sobre a prática do enfermeiro neste cenário. 0 enfermeiro deve ser capaz de avaliar 0 uso das tecnologias quanto aos aspectos de segurança, efetividade, custo benefício, impacto social, com ênfase na análise dos aspectos éticos envolvidos nas diferentes situações, a fim de que possa tomar decisões que favoreçam prioritariamente os interesses do paciente e não outros, de qualquer natureza. Considera-se que o grande desafio, na atualidade, seja preparar profissionais de forma a garantir a atualização e incorporação de conhecimentos necessários para o uso dos recursos tecnológicos existentes, em velocidade que os aproxime das máquinas, sem que se percam valores humanos fundamentais.

Palavras-chave: Recursos tecnológicos; Enfermagem oncológica; Bioética.

\section{Abstract}

This article has the purpose to discuss the technological advances incorporated in healthcare both, the hardware and software, that are important essential and complementary for the diagnosis and oncologic treatment; and to consider their implications on the nursing practice within this scenario. The nurse should be able to evaluate the use of these technologies on aspects regarding safety, effectiveness, cost-benefit, social impact, with emphasis on the analysis of the ethical issues surrounding the different situations. Therefore, the nurse can make decisions that favor, primarely, the patient's interests and not other interests, of any nature. It is considered that the great challenge in present times is to prepare professionals able to incorporate and update the knowledge necessary for the use of existing and new technologies without loosing the fundamental human values.
\end{abstract}

Key words Technological resources; O ncologic nursing; Bioethics.

${ }^{1}$ Enfermeira. Professora D outora do D epto de Enfermagem M édico-Cirúrgica da Escola de Enfermagem da USP.

${ }^{2}$ Enfermeira. Professora Associada do D epto de Enfermagem M édico-Cirúrgica da Escola de Enfermagem da USP.

Endereço para correspondência: Silvia Regina Secoli -Av Dr. Enéas de Carvalho Aguiar, 419 Cerqueira Cesar - São Paulo - SP Caixa Postal 41633 - CEP 05403-000 Fax (11) 3066-7546 


\section{INTRODUÇÃO}

0 desenvolvimento científico-tecnológico, verificado nas últimas décadas, na área de oncologia, promoveu considerável incremento no campo do diagnóstico e do tratamento, tendo-se, atualmente a cura como objetivo em cerca de $50 \%$ dos cânceres diagnosticados. ${ }^{1}$

Câncer é o termo aplicado a um conjunto de mais de 100 doenças, que se caracterizam pelo processo desordenado de multiplicação celular e capacidade de invasão para outras regiões e tecidos. 0 processo fisiopatológico inicia-se, quando uma determinada população de células sofre tranforformações no material genético contido no núcleo (ácido desoxirribonucléico - DNA), dando origem a descendentes insensíveis aos mecanismos reguladores do crescimento e divisão celular normal. 2,3 Logo, o câncer não é uma doença homogênea, trata-se de uma enorme variedade deformas de câncer, que acometem pessoas com qualquer idade, expostas a diferentes fatores de risco e que respondem de distintas maneiras aos inúmeros tratamentos, sendo, portanto, difícil englobar em um único termo o universo dos acontecimentos relativos a essa doença. ${ }^{4}$

Por causa dessa diversidade biológica, há um descompasso nos avanços relativos à terapia do câncer. Para alguns tipos, 0 arsenal medicamentoso disponibilizado no mercado tornou revolucionário o tratamento, possibilitando o aumento da taxa de cura, a sobrevida e a melhora da qualidade de vida. Entretanto, para outros, os avanços foram lentos e insuficientes para deter a malignidade da doença, ocorrendo o fracasso terapêutico. Porém, nos últimos anos, parte expressiva dos conhecimentos acumulados ocorreram graças ao incremento tecnológico.

D iante do exposto, a proposta do presente artigo foi tecer algumas considerações sobre as incorporações tecnológicas que revolucionaram o tratamento do câncer, e refletir sobre a prática do enfermeiro nesse cenário.

\section{TECNOLOGIA: UMA ABORDAGEM PARA A ONCOLOGIA}

Aurélio $0^{5}$ conceitua tecnologia como 0 "conjunto de conhecimentos, especialmente princípios científicos, que se aplicam a um determinado ramo de atividade". Para $\mathrm{M} \mathrm{cC}$ onnel ${ }^{6}$ tecnologias médicas são "técnicas, drogas, equipamentos e procedimentos usados pelos profissionais em saúde na assistência ao indivíduo e no sistema de saúde". Assim, falar em tecnologia é ter como referência a temática do trabalho. Segundo M erhy et al. ${ }^{7} 0$ trabalho em saúde atua distintamente de outros processos produtivos, em que os equipamentos e saberes tecnológicos estruturados dão a tônica do ritmo a ser seguido, pois o objeto - produção do cuidado - não é plenamente estruturado, e suas tecnologias de ação mais estratégicas se configuram em processos de intervenção em ato, uma vez que operam como tecnologias de relações, e encontros de subjetividade, para além dos saberes tecnológicos estruturados. $\mathrm{N}$ a enfermagem, as tecnologias são expressas na preocupação integral com a saúde (necessidades físicas, psicológicas, sociais, espirituais), na sistematização dos cuidados, na utilização do método clínico e na educação de familiares e cuidadores. ${ }^{8}$ A tecnologia representa a ação que um profissional desempenha sobre um indivíduo, com ou sem ajuda de instrumentos, com o objetivo de realizar alguma mudança nestse indivíduo. N essas acepções, a tecnologia é uma forma de expressão social e cultural, proporcionada pelas inovações e aplicações de pesquisas científicas. Por conseguinte, o trabalho em saúde é centrado nas pessoas, cujas capacidades de criação possibilitam diferentes formas para se produzir o cuidado.

Amara ${ }^{9}$ classificou as tecnologias envolvidas no trabalho em saúde em hardware e software. Em relação ao significado, esta categorização é muito próxima àquela realizada por $\mathrm{M}$ erhy, ${ }^{7}$ que ordenou as tecnologias em saúde em dura, leve-dura e leve.

$\mathrm{N}$ as tecnologias hardware ou dura são incluídos os artefatos de uso terapêutico como os equipamentos, produtos químico-farmacêuticos, procedimentos médico-cirúrgicos e dispositivos de uso terapêutico.7,9 As tecnologias software ou leve são constituídas por instrumentos sociais, que utilizam tecnologias de relações como produção de vínculo, acolhimento eo cuidado. 7,9,10

Segundo os conceitos apresentados, a oncologia tem incorporado tecnologias hardware, pode-se dizer - de ponta - como forma de proporcionar aos pacientes melhorias de qualidade e expectativa de vida. $\mathrm{N}$ o campo do diagnóstico, o incremento tecnológico tem possibilitado deteç̧ão precoce e monitoração da evolução dos tumores (TU) de forma mais acurada, permitindo melhor direcionamento do tratamento.

\section{Tecnologias hardware}

D entre os grandes responsáveis por esses avanços, destacam-se as pesquisas na área da genética e biologia molecular aliadas ao desenvolvimento da indústria químico-farmacêutica. O s novos medicamentos, especialmente os modificadores de resposta biológica (M RB), têm contribuído nas fases do diagnóstico e tratamento do câncer.

O s M RB são substâncias imunologicamente ativas 
que podem ter ação antitumoral direta. Esses agentes incluem as interleucinas, os interferons, os fatores estimulantes de crescimento de colônias, os anticorpos monoclonais, o Bacilo Calmette-Guerin (BCG), o levamisol e os indutores de diferenciação celular. ${ }^{11,12}$ D entre essas, o destaque é para os anticorpos monoclonais, que representam o grupo mais variado e bem estudado dentre os produtos biotecnológicos, além de serem, atualmente, a grande esperança terapêutica para muitos tipos de tumor.

No campo do diagnóstico, o desenvolvimento da tecnologia radiológica permitiu imagens de alta resolução e clareza. Entre os novos exames usados no campo do diagnóstico destacam-se PET (Positron Emission Tomography); ressonância magnética nuclear; SPECT (SinglePhoton Emission), tomografiaa qual mapeia funções fisiológicas no momento em que elas estão acontecendo. A combinação dessa tecnologia à informática permitiu 0 desenvolvimento das técnicas 3-D (Three Dimensional I maging), que combina imagens obtidas por outras técnicas e o CAD (Computer Aided D iagnosis) para análise de radiografias. A imunocintilografia é uma outra tecnologia sofisticada, que utiliza anticorpos monoclonais associados a isótopo radioativo para permitir mapeamento de metástases em todo 0 corpo. ${ }^{13}$

No campo da cirurgia oncológica, as novas técnicas como videoscopias; radiocirurgia esterotáxica; cirurgia utilizando laser de $\mathrm{CO}_{2}$, argônio; criocirurgia $\left(\mathrm{CO}_{2}\right.$ ou nitrogênio); biopsia do linfonodo sentinela; perfusão extracorpórea (isolamento de territórios tumorais e administração contínua de antineoplásicos); instalação de cateteres venosos ou arteriais, possibilitaram aos profissionais a real ização de intervenções menos invasivas, e aos pacientes benefícios no que concerne aos riscos de complicações e tempo de hospitalização. ${ }^{13-15}$

Em relação à radioterapia, a evolução da física e da eletrônica permitiram o desenvolvimento deaceleradores lineares de fótons e elétrons, de alto poder de penetração nos tecidos, dotados de sistemas comandados para 0 posicionamento do paciente e direcionamento do feixe de irradiação por 1, 2 ou mais pontos de entrada. M aior eficácia e acuidade (com maior preservação dos tecidos adjacentes ao tumor) têm levado a substituição progressiva da bomba de cobalto. ${ }^{16}$

$\mathrm{N}$ os cuidados paliativos, os principais avanços foram na área do alívio da dor. Procedimentos como a neuroablação; colocação de bombas de infusão de anal gésicos no tecido subcutâneo, e implante de cateteres venosos possibilitaram que o paciente com câncer pudesse ser assistido no domicílio, com conforto e melhora da funcionalidade. Além disso, substâncias como os hidrolóides, a papaína em gel e o carvão ativado, revolucionaram o conceito de curativo e 0 tratamento de feridas neoplásicas.

Freqüentemente, a tecnologia hardware é complexa, onerosa, limitada a um pequeno número de indivíduos e está sob controle de profissionais altamente especializados, qos quais se encontram em serviços sofisticados.

\section{Tecnologias software}

As tecnologias software, também, são amplamente adotadas, especialmente pela enfermagem, quetem como foco principal o cuidado e a manutenção da qualidade de vida do paciente. Elas são utilizadas em todas as fases do processo da doença, ou seja, diagnóstico, tratamento, reabilitação e quando os pacientes encontram-se fora de possibilidades terapêuticas. Esse tipo de tecnologia ajuda a resgatar aspectos da humanização, perdidos na segunda metade do século XX, quando a medicina apresentou um salto quântico no aspecto científicotecnológico, ${ }^{17}$ pois tem nos indivíduos - profissionais, paciente, família - os instrumentos de cuidado, responsáveis pelas transformações no estado de saúde. D entre as estratégias usadas destacam-se as cognitivocomportamentais, os métodos físicos e as técnicas de orientação educativa.

As técnicas cognitivo-comportamentais (TCC) são utilizadas em oncologia, principalmente, para controlar sintomas como a dor, o medo e a ansiedade. Os princípios norteadores do seu uso são de que os comportamentos humanos são socialmente aprendidos, e reforçados ou não, pela interação com o meio ambiente, pois o indivíduo não é receptor passivo de informações, e pode aprender ou reaprender comportamentos mais adaptativos, quelhe tragam maior funcionalidade e bem-estar. ${ }^{18,19}$

$\mathrm{N}$ as TC C, agrupam-se intervenções de análise e reorganização do comportamento e pensamento, como os trabalhos em grupos, e de aquisição estratégia para alívio do medo e ansiedade, tais como o uso de técnicas de relaxamento, distração e imaginação dirigida. Assim, o paciente reflete sobre os efeitos da doença na sua vida; aprende estratégias para 0 enfrentamento das situações estressantes, e participa do estabelecimento das metas que se quer alcançar. ${ }^{18-20}$

A atuação educativa do enfermeiro objetiva tornar os pacientes e familiares agentes de auto-cuidado e participantes ativos do processo terapêutico. As estratégias educativas são realizadas por meio de 
consultas individuais, discussões em grupos, demonstração, filmes, fitas cassetes, CD -RO M , folhetos educativos, manuais, entre outros, e podem ser feitas em diferentes contextos, incluindo o domicílio. ${ }^{19,20}$

Os métodos físicos mais utilizados pela equipe de enfermagem compreendem o uso de massagens e a aplicação de calor e frio superficiais. Há uma crença de que as técnicas que utilizam as mãos, reforçam a confiança do paciente, especialmente em situações de vida limítrofes. $\mathrm{N}$ a massagem manual, a aplicação de toque suave ou de força em tecidos moles proporciona alívio da dor e da tensão. É um método intuitivo, de prática muito antiga, que melhora a circulação local, relaxa a musculatura, traz uma sensação de conforto e bem-estar ao paciente e alivia a tensão psíquica. ${ }^{19,20}$

0 calor e o frio interferem no fluxo sangüíneo e metabolismo local, podendo aliviar certas condições álgicas. A aplicação de bolsas de água quente ou compressa parecem minimizar a dor, por causarem redução da isquemia tecidual, alívio do espasmo muscular e melhora do processo inflamatório local. Em contrapartida, o efeito analgésico do frio, obtido por meio do uso de bolsas de água fria, imersão em água fria, relaciona-se a vasoconstrição, e a redução da velocidade de condução de estímulos nervosos. ${ }^{19,20}$

A finalidade principal dessas técnicas apresentadas é ajudar a melhorar o funcionamento físico e psíquico do indivíduo, e reduzir a utilização dos serviços de saúde. Sabe-se que o câncer é uma doença que interfere em diversos domínios da vida - físico, emocional, social e espiritual - e que a interferência em algum deles pode modificar a apreciação da experiência global. 19,20 $^{2}$

Assim, do conjunto dos recursos tecnológicos disponíveis, constata-se que as tecnologias software são, em sua maioria, de baixo custo e fácil aplicação. M uitas são ensinadas aos pacientes e aos seus familiares para uso domiciliar, de modo seguro e eficiente, pois o risco de complicações e efeitos adversos é pequeno ${ }^{19}$, o que faz delas recursos acessíveis a um maior número de doentes portadores de câncer.

\section{O ENFERMEIRO E OS RECURSOS TECNOLÓGICOS NA ONCOLOGIA: UMA REFLEXÃO CRÍTICA.}

Pode-se dizer, que nas últimas décadas, a prestação de cuidados ao adulto com câncer tem sido fortemente influenciada pela explosão tecnológica, especialmente do tipo hardware. Porém, saber quantificar o tamanho ou a redução de um tumor, para a indicação do melhor tratamento, é tão relevante quanto avaliar corretamente o estado de ânimo de um pacientee ajudá-lo a sefortalecer para 0 enfrentamento da doença. Andrade $\mathrm{e}^{21}$ reflete sobre a atenção ao paciente com câncer e afirma que: "não há quimioterapia mais eficiente do que uma palavra amena, nem radiações mais benéficas do que 0 toque de uma mão que afaga. 0 médico em si pode ser um grande remédio, mas nem ele, nem seu arsenal terapêutico de drogas e radiações podem ser mais eficazes que o potencial curativo do próprio paciente".

Assim, a despeito de serem pouco discutidas e valorizadas na prática clínica, as tecnologias software tornaram-se fundamentais no cuidado do paciente com câncer, especialmente nos dias de hoje, em que as relações entre estado anímico e progressão do tumor são melhores compreendidas. ${ }^{21}$

Além do mais, não se pode analisar a explosão tecnológica como uma variável independente; é fundamental compreender de fato as repercussões no cuidado, especialmente no cenário brasileiro, em função das desigualdades sócio-econômicas da população, e os diferentes níveis de formação dos profissionais, que utilizam 0 arsenal tecnológico.

A tecnologia pressupõe uma preparação prévia dos profissionais que irão utilizá-la, no intuito desse recurso ser um veículo e não um fim em si mesmo. N esse contexto, é importante que os enfermeiros participem, ativamente, do processo de atualização científicotecnológica para terem subsídios que auxiliem no planejamento, organização, condução e avaliação desses recursos disponibilizados para o cuidado.

É fundamental, também, uma compreensão do uso das tecnologias, de maneira crítica, pois muitos dos recursos tecnológicos disponibilizados são considerados bens de consumo, geradores de lucros expressivos, para empresas e para profissionais. A ausência do aspecto crítico pode levar a incorporação irrestrita e desnecessária dos recursos tecnológicos, e, especialmente, a perda da autonomia na seleção das estratégias de interven ção. ${ }^{22}$

M uitos avanços tecnológicos resultam em criação denovos equipamentos enovas drogas, ao mesmo tempo em que trazem novas oportunidades e benefícios, acabam, também, por influenciar opções de escolhas que podem resultar em decisões equivocadas ou inadequadas. Aliás, o uso inadequado da tecnologia pode ser observado em diferentes situações, como por exemplo, na instalação um cateter em paciente que não tem condições de promover o auto-cuidado, ou mora distante de centros especializados para a sua manutenção; quando as chances de complicações superam os prováveis benefícios; quando reduzem a qualidade de vida do paciente e quando as mesmas metas podem ser 
obtidas por meios mais baratos, simples e básicos. ${ }^{22,23}$

$N$ esse sentido, os enfermeiros são essenciais no processo de tomada de decisão, podendo auxiliar clínicos, pacientes, familiares e serviços de saúde na escolha de produtos ou cuidados alternativos, independente do seu local de atuação. Também, no contexto hospitalar, no cuidado direto aos pacientes, questionamentos insistentes sobre a real necessidade do uso dos recursos tecnológicos, assim como da sua efetiva contribuição para a melhoria das condições de vida do paciente devem permear as atividades dos enfermeiros como integrantes da equipe multidisciplinar.

$\mathrm{N}$ a prática cotidiana, enfermeiros tendem a assumir a postura de que muitos equipamentos (bombas de infusão de analgésicos, monitores, entre outros) são confortáveis e seguros para os pacientes, além de aumentar a produtividade de seu trabalho. Entretanto, esquecem-se, muitas vezes, de avaliar, de fato, 0 benefício para o paciente. Em inúmeras situações, a atenção desse profissional volta-se para 0 alarme do equipamento, não para o chamado do paciente, desviando o foco principal do cuidado. Experiências têm apontado que pacientes, os quais utilizam dispositivos eletrônicos são menos avaliados pelos enfermeiros em relação à queixa de dor. ${ }^{23} \mathrm{~N}$ essas circunstâncias, em que pese 0 aumento da produtividade do trabalho do enfermeiro, na medida em que menor tempo é passado junto ao paciente, tem-se, em contrapartida, um distanciamento profissional que impossibilita outras estratégias de cuidados, inclusive da aplicação de tecnologia software.

A enfermagem é a equipe profissional que lida, de modo intensivo e integral, com as respostas dos pacientes, interagindo com seus sofrimentos, ansiedades e medos, em meio às estratégias terapêuticas. Assim, a despeito das ofertas tecnológicas do tipo hardware, é na tecnologia do tipo software que o trabalho da enfermagem encontra-se alicerçado. Todavia, deve haver em relação aos pacientes com câncer, um entendimento de que 0 curar, muitas vezes, é escravo da tecnologia, mas o cuidar aceita que a existência é finita e sempre existe algo que possa ser feito para melhorar a vida que resta. ${ }^{24-26}$

$\mathrm{H}$ ellengers, um dos fundadores do Instituto K ennedy de Bioética afirma que: "perto do fim da vida, uma pretensa cura representa, simplesmente, a troca de uma maneira de morrer pela outra". Afirma, ainda, que os profissionais devem dar mais atenção ao doente e menos à cura, visto que, em muitas vezes ela não ocorre. Assim, uma das principais ações na prática cotidiana da enfermagem, junto aos pacientes com câncer é acrescentar vida aos anos a serem vividos e não acrescentar anos à vida. ${ }^{24}$

$\mathrm{N}$ esse cenário, o enfermeiro deve ser capaz de avaliar 0 uso da tecnologia quanto aos aspectos de segurança, efetividade, custo benefício, impacto social, com ênfase à análise dos aspectos éticos envolvidos nas diferentes situações, ${ }^{3}$ a fim de que possa tomar decisões que favoreçam, prioritariamente, os interesses do paciente e não outros, de qualquer natureza.

D iante das inúmeras possibilidades existentes para a assistência dos pacientes oncológicos, a análise dos aspectos éticos inerentes ao uso dos diferentes recursos tecnológicos pode contribuir de maneira expressiva no direcionamento das tomadas de decisões técnicas, elevar em conta resultados desejáveis do ponto de vista holístico. 0 respeito aos princípios éticos da autonomia, beneficência, não-mal eficência ejustiça constitui suporte valioso para o alcance desse objetivo.

A autonomia, enquanto liberdade de escolha do indivíduo, é um dos valores mais importantes na vida cotidiana e, na vigência do $C A$, é um dos primeiros aspectos que o paciente perde ou vê afetado. Ele tem 0 direito de saber sobre sua doença, seu tratamento e participar das decisões, especialmente, quando estas são relacionadas a sua qualidade de vida. ${ }^{27}$ Pelo fato da autonomia ser afetada por vários fatores, internos e externos a sua pessoa, os enfermeiros devem ser sensíveis ao estado de vulnerabilidade do paciente e, ter a responsabilidade de não explorar essa condição, advogando no sentido defendêlo e protegêlo, inclusive contra a indicação e uso de tecnologia desnecessária. ${ }^{28}$

0 cuidado prestado por enfermeiros deve, na medida do possível, trazer benefícios aos pacientes e, em contrapartida, evitar possíveis danos não só em relação aos efeitos colaterais dos novos medicamentos, como também das indicações de tratamentos que pouco ou nada contribuirão para a melhoria do estado de saúde. $\mathrm{N}$ essas circunstâncias, são condutas éticas dos enfermeiros e demais profissionais envolvidos no atendimento de portadores de câncer, reconhecerem que a não maleficência deve prevalecer sobre a suposta beneficência, quando as chances de sucesso do tratamento mostram-se esgotadas e que investir na preservação da dignidade do paciente com 0 melhor emprego possível da tecnologia software é o que de mais beneficente se pode fazer. ${ }^{24,28}$

É certo, porém, que o desafio colocado aos profissionais está em distinguir entre o mal necessário, que pode ser tolerado, e o mal desnecessário, que pode e deve ser evitado. N essas circunstâncias, como as 
decisões e ações dos enfermeiros em defesa dos pacientes são, sobretudo de natureza ética, a eles cabe rever seus próprios valores e crenças, discutir com outros profissionais as perspectivas de tratamento ainda possíveis, dialogar com o paciente e família sobre as possibilidades, a fim de que sejam redefinidas as diretrizes para um cuidado voltado ao conforto e diminuição do sofrimento. ${ }^{25,26}$

O utro princípio a ser considerado é o da justiça, que talvez seja o mais difícil de ser abordado, pois é sabido que, especialmente no contexto hospitalar a seleção de estratégias e protocolos terapêuticos estão, intrinsecamente, atrelados aos aspectos financeiros, seja do indivíduo, seja da instituição de saúde. Todavia, a compreensão sobre a existência real de recursos limitados para uma demanda de atendimento cada vez mais presente, reafirma a necessidade de discussão e posicionamento dos profissionais sobre quais tratamentos indicar - a quem, quando e onde. Em outras palavras, que tecnologias utilizar, de modo a favorecer da melhor maneira possível um maior contingente de pacientes. ${ }^{24,27}$

No que concerne ao princípio da justiça, é oportuno indagar até que ponto a tecnologia influencia na saúde, no intuito de se adquirir subsídios que embasem decisões melhor fundamentadas. Nessa direção, dados provenientes de estudos farmacoeconômicos ${ }^{29}$ sobre fatores determinantes da saúde e consumo de recursos mostram que a tecnologia é o fator que participa com menor percentual na saúde (11\%), a despeito de ser 0 que mais consome recursos ( $90 \%)$. Tais resultados, que acenam para a importância das tecnologias software, vêm, mais uma vez, reiterar a responsabilidade ética dos profissionais envolvidos na indicação e seleção do melhor tratamento para os pacientes portadores de câncer.

\section{CONSIDERAÇÕES FINAIS}

N os países em desenvolvimento, a velocidade com que as novas tecnologias são introduzidas na prática, encontra-se em descompasso com o preparo do profissional para utilizá-la de maneira adequada. Assim, o grande desafio, na atualidade, é preparar profissionais de forma a garantir a atualização e incorporação dos conhecimentos necessários para o uso dos recursos tecnológicos existentes, em velocidade que os aproxime das máquinas, sem que se percam valores humanos fundamentais.

Os benefícios proporcionados pelos avanços tecnológicos, principalmente hardware, no tratamento do câncer, são inquestionáveis e 0 avanço da ciência no sentido de desenvolver e disponibilizar novos arsenais terapêuticos só tende a crescer, trazendo perspectivas otimistas para o tratamento das doenças. Contribuirá para isso, entender que a tecnologia por si só, não é boa nem má; apenas deve ser adequadamente empregada, para o que não se pode prescindir dos aspectos éticos a ela inerentes.

Em síntese, a participação dos enfermeiros na assistência aos pacientes com câncer tem um âmbito de ação privilegiado junto ao paciente, família e equipe multidisciplinar. Contudo, para que essa atuação se efetive, para além do domínio técnico-científico, há que ter em conta que, em qualquer circunstância, a presença cuidadora da enfermeira, característica intrínseca da profissão, jamais deverá ser substituída pela tecnologia, hardware ou software, por mais avançadas que sejam.

\section{REFERÊNCIAS}

1. Instituto $N$ acional deC âncer; $M$ inistério da Saúde. Ações de enfermagem para o controle do câncer: uma proposta deintegração ensino-serviço. 2a ed. Rio dejaneiro (Brasil): IN CA; 2002.

2. D eVitaVT, H ellman S, Rosenberg AS. Cancer principles and practice of oncology. 5th ed. Philadelphia: LippincottRaven; 1997.

3. Louro ID, M elo M SV, Galeotti S. Conceitos gerais de genética molecular In: Louro ID, LerenaJúnior JC, M elo M SV, Ashton-prolla P, Conforti-Froes N. G enética molecular do câncer. M SG Produção Editorial; 2002. cap. 1, p.11-24.

4. Werebe D M . D epressão no câncer. In: Fráguas Júnior R; Figueiró JAB. D epressõesem medicina interna eem outras condições médicas: depressões secundárias. São Paulo: Atheneu. 2000. cap. 18, p.159-64.

5. Aurélio BuarquedeH olanda. N ovo Aurélio: 0 dicionário dalíngua portuguesa. Rio deJ aneiro: N ovaFronteira; 2002.

6. M CC onnell EA. Perioperative nurses' roles in managing new technology. AO RN J. 1994;60(3):815-27.

7. M erhy EE. Em busca de ferramentas analisadoras das tecnologiasem saúde ainformação eo diaadiadeum serviço, interrogando e gerindo trabal ho em saúde. In: M erhy EE, O nocko R. Agir em saúde: um desafio para o público. 2aed. São Paulo: H ucitec; 1997. cap. 3, p. 113-50.

8. Ceccim RB. A ciência ea arte de um saber-fazer em saúde. In: M eyer DE, Waldow VR, Lopes M J M. M arcas da diversidade: saberesefazeres da enfermagem contemporânea. Porto Alegre; 1998. cap. 5, p. 87-102.

9. Amara R. Some observations on the interaction of technology and society. Future. 1975;7(2):515-7.

10. Jones CB, Alexander JW. The technology of caring: a 
synthesis of technology and caring for nursing administration. N urs Adm Q . 1993;17:11-20.

11. LopesEB, Secoli SR. M odificadores da resposta biológica: noções para o planejamento das intervenções de enfermagem. Acta O ncol Bras. 1999;19(1):267-72.

12. Secoli SR, LopesEB. 0 monitoramento depacientesrecebendo interleucinaeinterferon: basesfármaco-dínicasparaa assistência deenfermagem. N ursing. 1999;2(8):30-4.

13. Giglio AD, Yamaguchi N H . D iagnóstico eestadiamento clínico do câncer. In: SociedadeB rasileira deC ancerologia. Curso básico de cancerologia. São Paulo: M SG ; 1999. cap. 4, p. 39-55.

14. Landreneau RJ. N ova técnica reduz riscos da cirurgia de câncer de pulmão. Prat H osp. 2001;3(13):62-4.

15. O liveiraFilho RS, Santos IAO, Ferreira LM . Perspectivas do linfonodo sentinela na cirurgia oncológica. Prat H osp. 1999;1(6):42-4.

16. FreitasPP. Princípios gerais da radioterapia. In: Sociedade Brasileira de $C$ ancerologia. C urso básico decancerologia. São Paulo: M SG ; 1999. cap. 7, p. 74-9.

17. Figueiredo M TA. Cuidados paliativos: respeito, alívio e dignidade para o paciente. Prat H osp. 2003;5(27):68-70.

18. Kingdon RT, Stanley KLJ, Kizior RJ. Cognitive behavioral therapy. In: $\mathrm{H}$ andbook for pain management. Philadelphia: W B Saunders; 1998. p. 101-15.

19. Pimenta CAM . D or oncológica: bases para avaliação e tratamento. M undo saúde. 2003;27(1):98-110.
20. M CC affery M , Pasero C . Pain: clinical manual. St Louis: M osby; 1999.

21. AndradeR. A atenção holística ao pacienteoncológico. Prat H osp. 2003;5(27):85-7.

22. Jennett $B$. H igh techonology medicine: how defined and how regarded. M ilbank $M$ Fund $\mathrm{Q} H$ ealth Soc. 1985;63(1):141-73

23. Ferrell BR, W hedon $M$. Professional and ethical considerations in the use of high-tech pain management. O ncol N urs Forum. 1991;18:1135-43.

24. Pessini L, De Barchifontaine CP. Problemas atuais de bioética. 6a ed. São Paulo: Loyola; 2002.

25. Pessini L. A filosofiados cuidados pal iativos: uma resposta diante da obstinação terapêutica. M undo Saúde. 2003;27(1):15-32.

26. M cC oughlan M. A necessidade de cuidados paliativos. M undo Saúde. 2003;27(1):6-14.

27. CostaSJF, GarrafaV, O selkaG . Iniciação a bioética. Braślia: Conselho Federal deM edicina; 1998.

28. Piva JP, C arval ho PRA. Consideraçõeséticas nos cuidados médicos do pacienteterminal. Bioética. 1993;1:129-38.

29. BevilácquaLD P. Farmacoeconomia In: GomesM JV M , Reis AM M . Ciência farmacêuticas: uma abordagem em farmácia hospitalar. São Paulo: Atheneu; 2001. cap. 11, p. 191-220.

30. D avisA J. Las dimensiones éticas del cuidar em enfermeria. Enferm Clín. 1999;9:21-34. 